\title{
Role of detection of lipoarabinomannan (LAM) in urine for diagnosis of pulmonary tuberculosis in HIV patients: Egyptian experience
}

\author{
Nagwa Elhalawany ${ }^{1}$, Nessrin Shalaby², Amal Fathy², Ahmed S. Elmorsy², Mohamed Zaghloul ${ }^{3}$, \\ Heba El-shahawy ${ }^{3}$ and Asem A. Hewidy ${ }^{2 *}$
}

\begin{abstract}
Background: Tuberculosis remains a worldwide problem fueled by the HIV epidemic. TB infection impacts HIV progression and mortality even with treatment. Egypt has increasing HIV prevalence, although still in low prevalent areas.

Results: Urinary LAM was positive in 22 (95.7\%) of TB patients and 1 (1.9\%) of non TB group. Sensitivity was 95.7\%, specificity $98.1 \%$, positive and negative predictive values were $95.7 \%$ and $98.1 \%$ respectively, with accuracy $97.4 \%$. Urinary LAM ELISA assay has the highest sensitivity (95.7\%) in relation to other tests used for TB detection in HIV patients and its concentration was highly correlated to CD4 cell count and the extent of radiological changes.

Conclusion: The use of urinary LAM in HIV patients is rapid, safe, available, and helpful tool for ruling in TB especially for those who cannot expectorate, critically ill, with low CD4, or presented by multiple system affection.
\end{abstract}

Keywords: Tuberculosis, HIV, Lipoarabinomannan (LAM)

\section{Background}

Mycobacterium tuberculosis infection clearly impacts HIV progression and mortality, and disease from $M$. tuberculosis increases in HIV-infected patients as CD4 cell counts decline [1].

However, the prevalence of HIV infection in Middle East and North Africa (MENA) MENA is $0.1 \%$ which is the lowest worldwide, HIV-related deaths were estimated to be 15,000 cases in 2013 which representing increase by two-thirds since 2005 [2].

Although marked progress in HIV management, late presentation of cases with TB still represents a major threat to survival. The lack of rapid and accurate assays for HIV-associated TB has been a major hindrance to

\footnotetext{
* Correspondence: ahewidy@yahoo.com

${ }^{2}$ Chest Medicine Department, Faculty of Medicine, Mansoura University,

Algomhoria Street, Mansoura City, Egypt

Full list of author information is available at the end of the article
}

reducing deaths from these diseases in resource-limited settings [3].

The aim of this study was to evaluate the role of urinary LAM in diagnosis of active tuberculosis in patients with HIV in relation to different diagnostic tools to help early management for improving outcome.

\section{Methods}

A diagnostic validity study in which urinary LAM was done in seropositive HIV patients with symptoms suggestive of TB who were included and investigated with battery of tests to reach the final diagnosis.

The study was conducted at Mansoura Fever Hospital in collaboration with the Chest Department and Clinical Pathology Department, Mansoura University, Egypt, from January 2015 to October 2016.

Ethical approval had been obtained from Medical Research Ethics Committee of Faculty of Medicine, Mansoura 
University (code no: MD/138). Approval from Egyptian Ministry of Health was taken (no.: 1-2016/18). The study was registered in clinical trial number (NCT04813666). Patients signed their written consents after detailed explanation of the study protocol.

This study started by 214 seropositive HIV adult patients from Mansoura Fever Hospital screened by symptoms suggestive of TB; only 76 patients of them were included due to presence of positive screening and the other 214 have been excluded because of negative screening for TB. The 76 participants were 62 (81.6\%) males and $14(18.4 \%)$ with mean age $36.4 \pm 9.5$ years, categorized into 2 groups: TB group included 23 patients (30.3\%) and non TB included 53 patients (69.7\%).

The study included seropositive HIV patients with a new diagnosis of either pulmonary or extra-pulmonary $\mathrm{TB}$, and also included patients who were presented by symptoms suggestive for tuberculosis, changes in chest $\mathrm{X}$-ray that was not improved by full course of antibiotics or positive tuberculin test $\geq 5 \mathrm{~mm}$.

Patients on anti-TB drugs or negative screening for sero-positive HIV patients were excluded from the study.

After patient acceptance to be included in the study, the patients were subjected to the following:

1- Detailed history taking: This included personal history, present history with stress on presence and duration of current cough, fever, weight loss and night sweats, past history of chest diseases or other diseases, family history, and history of contact with TB patient. The screening carried out in this study was as follows: current cough, fever, weight loss, or night sweats? Any positive answer was considered as positive screening and the patient was included [4].

2- Clinical examination: General examination and local examination of the chest had been done for all patients.

3- Radiological examination: CXR posteroanterior view had been done for all patients.

4- CD4 count has been done in Central lab of Cairo for 64 (84.2\%) of patients the missing 12 patients were unable to travel Cairo.

5- Tuberculin test TST, sputum ZN staining, and sputum culture on Löwenstein-Jensen medium.

6- Empirical therapy had been started for all patients according to the presentation of the patient. For example, patients with consolidation, empirical therapy was in the form of macrolide with third generation cephalosporin.

7- GeneXpert MTB/RIF was done for 42 sputum negative patients who were not responding initially on antibiotic empirical therapy.
8- Flexible bronchoscopy and bronchoalveolar lavage for 9 patients (8 in Mansoura Chest Hospital and one in Chest Department of Mansoura University Hospital) who were not responding to empirical therapy with negative sputum ZN and GeneXpert.

9- CSF examination for 5 patients those presented by symptoms and signs suggestive for meningitis (persistent headache, vomiting, fever, and neck rigidity) including biochemical, adenosine deaminase (ADA), and microbiological examination.

10- Paracentesis have been done for one patient that presented with ascitis in Tropical Department of Mansoura Univeristy and ascitic fluid analysis by cell count, albumin level, culture, total protein, Gram stain, ADA, and cytology.

11- Therapeutic test was applied for six patients those not responding to empirical with negative results and picture consistent with tuberculosis considered by the team of the work. The response was monitored by the clinical and radiological improvement. Using Isoniazid $5 \mathrm{mg} / \mathrm{kg}$ body weight+ rifampicin $10 \mathrm{mg} / \mathrm{kg}$ + pyrazinamide 25 $\mathrm{mg} / \mathrm{kg}+$ ethambutol $15 \mathrm{mg} / \mathrm{kg}$ in addition to vitamin B6 (pyridoxine) $25 \mathrm{mg}$ daily.

12- The tuberculous patients who were not admitted in fever or chest hospitals had been followed up monthly.

13- Urinary LAM ELISA: The test has been done in Clinical Pathology Department of Mansoura Faculty of Medicine using LAM ELISA kits of Sunred Biotechnology Company and the test had performed according to manufacturer's instructions [5].

Standard dilution was done according to the manufacture instructions. For example, to reach concentration of $1200 \mathrm{pg} / \mathrm{ml}$, we add $120 \mu \mathrm{l}$ standard $(2400 \mathrm{pg} / \mathrm{ml})$ to 120 $\mu \mathrm{l}$ standard diluents. Final measures in which blank well has been considered as zero, the optical density (OD) measured less than $450 \mathrm{~nm}$ wave length within $15 \mathrm{~min}$ after adding the stop solution. According to standards' concentration and corresponding OD values, a standard curve instituted by plotting OD for each standard on the $x$-axis against the concentration on the $y$-axis (Table 1 )

Table 1 Absorbance values (OD) against the standard concentrations

\begin{tabular}{ll}
\hline Standard concentration & Mean absorbance \\
\hline $2400 \mathrm{pg} / \mathrm{ml}$ & 2.061 \\
$1200 \mathrm{pg} / \mathrm{ml}$ & 1.417 \\
$600 \mathrm{pg} / \mathrm{ml}$ & 1.019 \\
$300 \mathrm{pg} / \mathrm{ml}$ & 0.634 \\
$150 \mathrm{pg} / \mathrm{ml}$ & 0.344 \\
\hline
\end{tabular}


then the most acceptable curve drawn along the points on the diagram paper. The compatible concentration then founded out as stated by the sample OD value by the sample curve.

Summary of the procedures: the steps are summarized as follow:

1- Preparing samples, reagents, and standards

2- Adding the antibodies labeled with enzyme, samples, and standards, incubating $1 \mathrm{~h}$ at $37^{\circ} \mathrm{C}$

3- Plate washing several times, adding chromogen solutions, and incubating $10 \mathrm{~min}$ at $37^{\circ} \mathrm{C}$

4- Adding the stop solution

5- Measure optical density in $10 \mathrm{~min}$

6- Calculation

14- Statistical analysis: The statistical analysis of data was done by SPSS program, version 22 (New York, USA). Qualitative data were analyzed as percentages and numbers. Fisher's exact and Monte Carlo tests were utilized for groups' comparison, accordingly. Quantitative data were analyzed as means or medians, according to the variant. They were tested for normality by Kolmogorov-Smirnov test. In the normally distributed variants, independent sample $t$ test and one way ANOVA were used for comparison between groups. In the nonnormally distributed variables, Mann-Whitney test was used for comparison between groups. Sensitivity, specificity, positive predictive value (PPV), negative predictive value (NPV), and accuracy were calculated to evaluate the efficiency of urine LAM value in the diagnosis of pulmonary TB. Correlation between two variables was done using Pearson's correlation. $P$ value $\leq 0.05$ was considered to be statistically significant.

\section{Results}

There were 214 people living with HIV (PLWH) screened for TB, 76 only were positive and included in the study. There were 138 PLWH with no symptoms, so they were excluded. The positively screened 76 patients (62 (81.6\%) males and 14 (18.4\%) females) were included and investigated for TB then categorized into 2 groups: TB group included 23 patients $(30.3 \%)$ and non TB included 53 patients $(69.7 \%)$.

TB group included 23 patients (19 males and 4 males) with mean age of $35.43 \pm 9.98$ years and median range of CD4 count ( 159 cells $/ \mu \mathrm{l})$ and non TB group included 53 patients (43 males and 10 females) with mean age of $36.83 \pm 9.35$ years and median range of CD4 (154 cells/ $\mu \mathrm{l})$. Both groups were age, sex, and CD4 count matched $(p>0.05)$ (Table 2).
The mean age of TB group was $36.4 \pm 9.5$ years and $36.83 \pm 9.35$ years for non TB group without significance. There were 19 (82.6\%) males among TB group and 43 $(81.1 \%)$ in non TB. On the other hand, females were 4 (17.4\%) and 10 (18.9\%) among TB and non TB respectively with no significant difference.

The median CD4 cell count was insignificantly higher among $\mathrm{TB}$ group than non $\mathrm{TB}$

\section{Radiological findings in both groups}

Upper, lower, and extensive abnormalities in TB group were $7(35.0 \%), 2(10.0 \%)$, and $11(55.0 \%)$ respectively, while were $3(15.0 \%), 12(60.0 \%)$, and $5(25.0 \%)$ in non TB group, the upper and extensive abnormalities were of high statistical significance $(p, 0.005)$ between the 2 groups (Table 3 ).

All patients in the TB group presented by radiological findings in the form of infiltrates 20 (100\%) while non TB group infiltrations presented in $17(85.0 \%)$ and 3 $(15.0 \%)$ of patients shown cavitations without statistical significance $(p, 0.231)$.

The 20 patients with abnormal radiology were diagnosed as community-acquired pneumonia, Pneumocystis carinii pneumonia, Cryptoccocus meningitis with CAP and lymphoma. Five patients were unexplained fever assumed to HIV after no improvement with therapeutic trial of antituberculous treatment.

Tuberculin test was positive only $2(8.7 \%)$ patients of TB group and all non TB was negative without statistical significance $(p, 0.089)$. The tuberculin test had sensitivity $8.7 \%$, specificity $100 \%$, PPV and NPV were 100\%, 71.6\% respectively with accuracy $72.3 \%$.

Positive direct sputum smears $(\mathrm{ZN})$ with positive sputum cultures were in 9 (42.9\%) patients, while ZN was negative with positive culture in $4(19 \%)$ patients and both were negative in $8(38.1 \%)$ of those that have produced sputum.

For patients with negative smears, GeneXpert was done; there were $4(33.3 \%)$ patients with positive Gene and culture, while Gene was positive in $4(33.3 \%)$ patients with negative culture. Both GeneXpert and culture were negative in 4 patients (33.3\%) that were received therapeutic test with the other 2 patients who have no sputum or BAL, all of them have shown initial clinical response on anti TB as illustrated in Fig. 1.

\section{Qualitative LAM analysis}

LAM was done for all patients; it was positive in 22 patients $(95.7 \%)$ of TB and one patient (1.9\%) of non TB group. The sensitivity was $95.7 \%$, specificity $98.1 \%$, positive and negative predictive values were $95.7 \%$ and $98.1 \%$ respectively, with accuracy $97.4 \%$ (Table 4 ). 
Table 2 Demographic data and CD4 characteristics of TB and non TB groups

\begin{tabular}{|c|c|c|c|}
\hline Characteristic & $\begin{array}{l}\text { TB group } \\
(N=23)\end{array}$ & $\begin{array}{l}\text { Non TB group } \\
(N=53)\end{array}$ & Test of significance \\
\hline $\begin{array}{l}\text { Age (years) } \\
\text { Mean } \pm S D\end{array}$ & $35.43 \pm 9.98$ & $36.83 \pm 9.35$ & $\begin{array}{l}T \text { test } \\
t,-0.585 \\
p \text { value, } 0.560\end{array}$ \\
\hline Gender & No. (\%) & No. (\%) & \multirow{3}{*}{$\begin{array}{l}\text { Fisher's exact test } \\
p \text { value, } 1.00\end{array}$} \\
\hline Male & 19 (82.6\%) & $43(81.1 \%)$ & \\
\hline Female & $4(17.4 \%)$ & $10(18.9 \%)$ & \\
\hline $\begin{array}{l}\text { CD4 (cells/Ml) } \\
\text { Median range (Q1-Q3) }\end{array}$ & $159(93.25-248.25)$ & $154(90-270.5)$ & $\begin{array}{l}\text { Mann-Whitney test } \\
Z_{1}-0.217 \\
p \text { value, } 0.828\end{array}$ \\
\hline
\end{tabular}

TB tuberculosis, SD standard deviation, CD4 cluster differentiation

\section{Quantitative LAM analysis}

The mean LAM concentration was $(474.77 \mathrm{pg} / \mathrm{ml} \pm$ 149.3) with very high negative significant correlation with CD4 count $(r,-0.88, p<0.001)$ (Table 5).

\section{Discussion}

$\mathrm{TB} / \mathrm{HIV}$ co-infection is known to have a wide variety of clinical manifestations and radiological findings mostly are atypical and/or non-specific, so TB diagnosis in those populations is considered a problematic dilemma [6].

Although TB-related deaths between HIV patients have decreased since 2004 by 32 percent, it is still the leading main cause of death among those people, as about one in three HIV-related deaths is caused by tuberculosis [7].

Although marked progress in HIV management, late presentation of cases with $\mathrm{TB}$ still represents a major threat to survival. Case fatality rates among people being treated for TB are more than three times greater if they are HIV-positive than if they are HIV-negative and even higher if they are not put on ART as soon as possible after starting TB treatment [8].

Table 3 Presence of abnormality, site, and pattern of CXR abnormalities in TB and non TB groups

\begin{tabular}{|c|c|c|c|}
\hline Characteristic & $\begin{array}{l}\text { TB group } \\
(N=20) \\
\text { No. }(\%)\end{array}$ & $\begin{array}{l}\text { Non TB group } \\
(N=20) \\
\text { No. }(\%)\end{array}$ & Test of significance \\
\hline Abnormal & $20(86.9 \%)$ & $20(37.7 \%)$ & \multirow{2}{*}{$\begin{array}{l}\text { (Fisher's exact test) } \\
p<0.001\end{array}$} \\
\hline Normal & $3(13.1 \%)$ & $33(62.3 \%)$ & \\
\hline \multicolumn{3}{|l|}{ Site } & \multirow{4}{*}{$\begin{array}{l}\text { (Monte Carlo test) } \\
p 0.005^{*}\end{array}$} \\
\hline Upper lobe* & 7 (35.0\%) & $3(15.0 \%)$ & \\
\hline Lower lobe & $2(10.0 \%)$ & $12(60.0 \%)$ & \\
\hline Extensive* & $11(55.0 \%)$ & $5(25.0 \%)$ & \\
\hline \multicolumn{3}{|l|}{ Pattern } & \multirow{3}{*}{$\begin{array}{l}\text { (Fisher's exact test) } \\
\text { p } 0.231\end{array}$} \\
\hline Infiltrate & $20(100 \%)$ & 17 (85.0\%) & \\
\hline Cavity & $0(0 \%)$ & $3(15.0 \%)$ & \\
\hline
\end{tabular}

${ }^{*} P<0.001$ : high significance
The aim of this study was to evaluate the role of urinary LAM in diagnosis of active tuberculosis in patients with HIV in relation to different diagnostic tools to help early management for improving outcome.

\section{Clinical screening for active TB among PLWH}

While [9] concluded that symptom-based screening with smear microscopy was insufficiently sensitive and more sensitive diagnostic testing is required for HIV patients, where the screening had $54 \%$ sensitivity much lower than this study $(82.6 \%)$, also, there were five $(9.25 \%)$ of the 54 participants with $\mathrm{TB}$ had none of the cardinal symptoms of TB.

According to WHO, 2011, all PLWH should be screened for TB by symptom-based screening as guidelines states that PLWH who not presented by current cough, fever, weight loss, or night sweat are rarely have active TB, while those who have any one symptom may have active TB and should be evaluated for TB or other diseases [4].

\section{Radiological findings in both groups}

As expected, a large percent of TB patients were presented by bilateral extensive infiltrates while 3 patients (13\%) were of no apparent CXR abnormalities, with sensitivity (87\%) and (62.3\%) specificity. This was comparable to [10] who found limitation of radiological abnormalities consistent with TB where the sensitivity (68\%) and the specificity (53\%).

Despite relative low specificity, chest radiology had more sensitivity than direct ZN smear (87\% vs. $42.8 \%$ ) in this study also; it helps rapid assessment of patient especially during the first visit to health care providers. Chest radiography was found to improve the screening sensitivity when added to clinical screening for TB in PLWH [4].

\section{Tuberculin test}

Although cutoff point for TST was $5 \mathrm{~mm}$ for those patients, it was positive only in 2 patients of TB group $(8.7 \%)$, the reduction in sensitivity of the TST is 


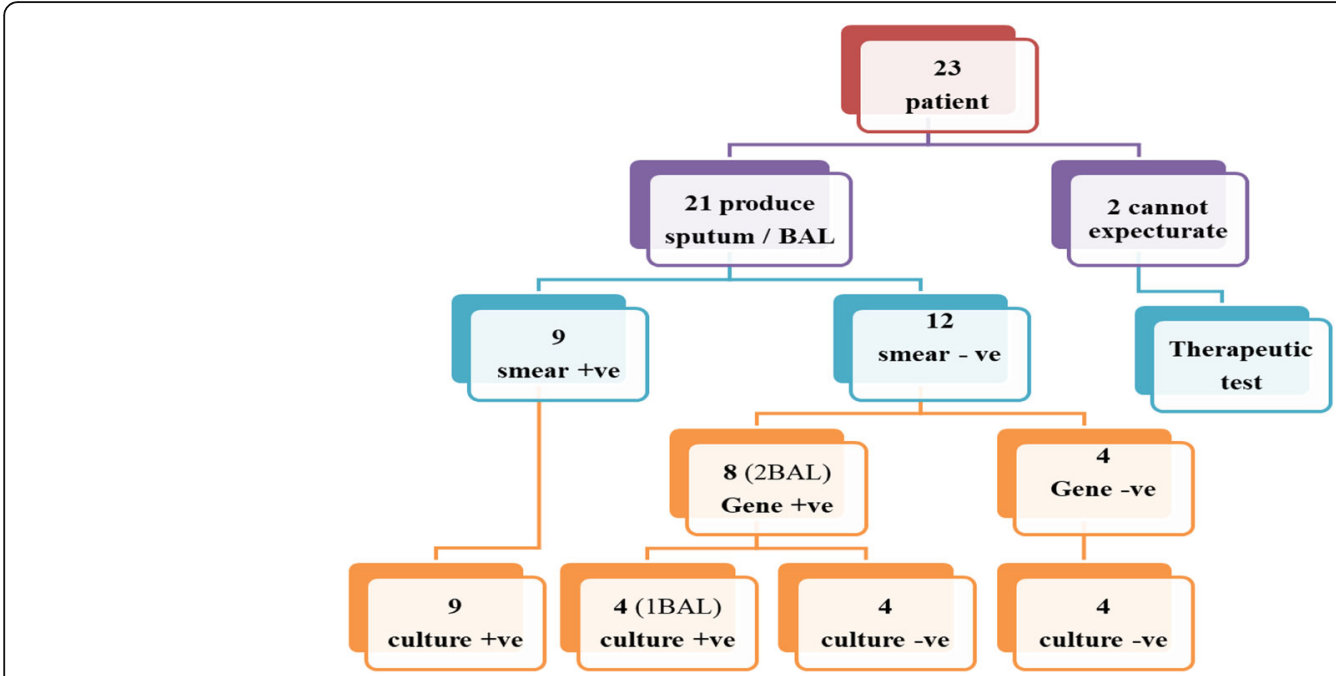

Fig. 1 Flowchart for TB diagnosis in TB group

predominantly caused by anergy that occurs as an all-ornon phenomenon. Consequently, the gain in sensitivity of reducing the cutoff value for a positive TST result from $10 \mathrm{~mm}$ to $5 \mathrm{~mm}$ is limited [11].

\section{Pulmonary with extrapulmonary TB (EPTB)}

Six TB patients (26\%) were presented in our study by pulmonary and extra pulmonary $\mathrm{TB}$, apparently 5 patients of them had meningitis and one patient had peritonitis, as all these patients were severely ill with very low CD4. This finding was comparable to [12] who found that patients co-infected by HIV and TB who had low CD4 cell counts were more susceptible to TB meningitis than others. We had detected no cases with pleural disease which was comparable to [12, 13] who concluded that HIV patients with EPTB were less likely to develop TB pleurisy when compared to patients without HIV.

\section{Qualitative urinary LAM}

Urinary LAM assay in our HIV patients had higher sensitivity, specificity, positive and negative predictive values (95.7, 98.1, 95.7 and 98.1) respectively as only one patient of TB group was negative who presented by only cough (without sputum), CD4 $=485$ cells $/ \mathrm{mm}^{3}$, normal

Table 4 Results of LAM test in both groups (Qualitative LAM analysis)

\begin{tabular}{|c|c|c|c|}
\hline LAM & $\begin{array}{l}\text { TB group } \\
N=23 \\
\text { No. (\%) }\end{array}$ & $\begin{array}{l}\text { Non TB group } \\
N=53 \\
\text { No. }(\%)\end{array}$ & $\begin{array}{l}\text { Total } \\
N=76 \\
\text { No. (\%) }\end{array}$ \\
\hline Positive & $22(95.7 \%)$ & 1 (1.9\%) & $23(30.3 \%)$ \\
\hline Negative & $1(4.3 \%)$ & 52 (98.1\%) & 53 (69.7\%) \\
\hline
\end{tabular}

chest X-ray, positive TST, negative smear, and culture while positive GeneXpert, clinically consistent with latent tuberculosis.

The only false positive result was for patient presented by lymphoma (generalized lymphadnopathy diagnosed in oncology center as HIV-related lymphoma-not specified).

The false positive LAM antigen could be apparent false result due to undetected active tuberculosis anywhere as it is released only from degenerated or active TB bacilli or it may be true false positive as a result of urinary contamination by non-tuberculous strains although it is usually of inadequate concentration to cross-react with LAM Ag test. It was demonstrated that there is very low cross-reactivity between tuberculous and non-tuberculous LAM antigen [14].

While blood, bacterial, or fungal contamination shows no effect on ELISA results, dust and soil may cause increased OD (optical density) giving rise to false positive ELISA results for LAM. So, urine collection for LAM testing should be under strict conditions to avoid the contamination [15].

Urinary LAM released by MTB bacilli may be produced within the urinary tract or released from other infected sites dependent on to what extent this LAM is present in the free form (uncomplexed by immune response) in the circulation to be filtered in urine [16].

Table 5 Correlation between LAM concentration and CD4 count

\begin{tabular}{lllc}
\hline CD4 (median & LAM & \multicolumn{2}{l}{ Pearson correlation } \\
\cline { 3 - 4 } $\begin{array}{l}\text { Q1-Q3)) } \\
N=16\end{array}$ & $\begin{array}{l}\text { concentration } \\
\text { Mean }(\mathbf{p g} / \mathbf{m l}) \pm \text { SD } \\
\boldsymbol{N}=\mathbf{2 2}\end{array}$ & $\boldsymbol{r}$ & $\boldsymbol{p}$ \\
\hline $159(93.25-248.2)$ & $474.7 \pm 149.3$ & -0.889 & $p<0.001^{*}$ \\
\hline
\end{tabular}

CD4 cluster differentiation, LAM lipoarabinomannan ${ }^{*} P<0.001$ : high significance 
Is urinary lipoarabinomannan the result of renal tuberculosis? [17] have tried to answer by renal pathological examination in $36 \mathrm{HIV}$-infected autopsies from Ugandan and conclude that positive LAM results explained by urinary tuberculosis in the majority of cases, and cases with disseminated tuberculosis without renal affection also diagnosed by LAM (as 5 positive LAM patients (38\%) had disseminated disease without renal involvement). This result has suggested other mechanisms that cause LAM antigenuria may take place in HIV patients.

However, the proportion of LAM in urine is originated from urinary tract tuberculosis or resulted from pulmonary TB; this has no impact on the specificity of the test, as extra-pulmonary tuberculosis is most frequently associated also with pulmonary in HIV patients [18].

HIV patients with tuberculosis may be missed by sputum-based tests for many reasons: low sputum bacillary load that is typically seen with them; they may not be able to provide sufficient and/or high quality sputum samples or even no sputum at all; in addition to increased extrapulmonary tuberculosis proportion in PLWH [3].

Tests based on urine would have advantages over that based on sputum as urine is easy to collect and store, and also, is lacking the infection risks associated with sputum examination.

Several hypotheses have explained the higher sensitivity of urinary LAM in HIV patients especially in late stages, including high TB bacillary burden, so higher antigen load, increased incidence of genitourinary tuberculosis, and increased glomerular permeability to permit higher urinary antigen levels. An alternative hypothesis is that MTB bacilli are less able to be anatomically compartmentalized at low level of CD4 counts, which leading to increased risk of disease dissemination [19].

The sensitivity and specificity $(95.7,98.1)$ of urinary LAM in this study were comparable to many studies that had evaluated urinary LAM ELISA assay (using sputum culture as the gold standard test). The main common observation was that the sensitivity is very significantly correlated to CD4 count and the specificity ranging from 96 to $100 \%[3,20]$.

Also, the findings were comparable to [21] who found the sensitivities of the urinary LAM test and Xpert test (that was applied to sputum or urine samples) were greatly higher than sputum smear (25\% of patients were sputum positive) or radiology (one quarter have no radiological abnormalities). The sensitivity of urinary LAM reached $100 \%$ in those died within 3 months of diagnosis.

While our study was incomparable to [22] who concluded that urine LAM assay is an imperfect pathogen biomarker for an active TB infection, but might provide additional diagnostic value in certain clinical settings. However, with a peak sensitivity of only $68 \%$ among the most severely ill patients and urine LAM test should not be used to rule out active TB disease, the LAM high band intensity (by strip test) had a diagnostic specificity of $100 \%$, or no false positive results, the presence of a strong LAM band intensity provides good diagnostic value to clinicians.

Urine LAM considered being of superior ruling in tool when compared to empiric therapy among HIV inpatients in countries with a lower TB prevalence due to lack of standardization of empiric treatment decisionmaking. This superiority is also manifested when compared it to the poor diagnostic utilities especially in PLWH with advanced disease [23].

Also, [24] concluded that the urinary LAM was detected over $50 \%$ of TB suspect cases with advanced HIV and when this test used in combination to the direct sputum smear, they detected two-thirds of TB cases. The limitation of that study was overestimation of the true sensitivity or underestimation of the true specificity of the urinary LAM by including sputum and blood cultures as standard tests that might have failed to detect some TB cases as solely extrapulmonary, nondisseminated tuberculosis.

WHO policy recommendations in 2015 for the usage of the lateral flow urine lipoarabinomannan (LF-LAM) test, also, matched with these findings as the policy recommended the use of LF-LAM in TB diagnosis only for PLWH with low CD4 counts (less than or equal to 100 cells $/ \mu \mathrm{L}$ ) or who are seriously ill (defined based on 4 danger signs: respiratory rate $>30 / \mathrm{min}$, temperature > $39{ }^{\circ} \mathrm{C}$, heart rate $>120 / \mathrm{min}$ and unable to walk unaided) regardless of CD4 count or with unknown CD4 count out or inpatients [25].

\section{Quantitative urinary LAM}

The mean LAM concentration was $474.77 \mathrm{pg} / \mathrm{ml} \pm 149.3$ with very high negative significant correlation with CD4 count $(p<0.001)$, and high significant correlation with site of radiological changes $(p, 0.001)$ where there were very high negative correlation with upper changes $(p<$ $0.001)$ and very high positive correlation with extensive changes $(p<0.001)$.

Whether the patient with lymphoma with positive high LAM concentration was true false positive, misdiagnosed, or has both $\mathrm{TB}$ and lymphoma, we cannot answer.

The exact diagnosis of lymphomas appeared to be challenging for pathologists, even in developed countries without hematopathological specialization. Changing diagnosis was made in 20\% (reaching $44 \%$ in developing one) of cases submitted to have lymphoma in the UK after central review [26].

These results were also comparable to [27] who confirmed a significant difference in median OD for those 
with more localized disease in relation to those with disseminated disease $(p<0.001)$. Also, significant negative correlation between the urine LAM test OD and CD4 count.

Use of quantitative urinary LAM results allowed additional clinical data about the degree of TB severity that consequently reflected on the outcome of cases could not be obtained from qualitative results alone. Quantitative analysis of LAM might thus offer a more complete understanding of test performance and the best test usage. Clinical studies have shown that quantitative test results were positively related to increasing bacillary burden [28].

\section{Conclusion}

The use of urinary LAM in HIV patients is rapid, safe, available, and helpful tool for ruling in TB especially for those who cannot expectorate, critically ill, with low $\mathrm{CD} 4$, or presented by multiple system affection.

\section{Abbreviations}

HIV: Human immunodeficiency virus; TB: Tuberculosis;

LAM: Lipoarabinomannan; MENA: Middle East and North Africa;

PLWH: Patients living with HIV

\section{Acknowledgements}

None.

\section{Authors' contributions}

All authors have read and approved the manuscript. NI had made substantial contributions to the conception and had approved the submitted version and had agreed both to be personally accountable for the author's own contributions. NS and AF had done design of the work and had approved the submitted version and had agreed both to be personally accountable for the author's own contributions; MZ and HE had the acquisition, analysis, and interpretation of data and had approved the submitted version and had agreed both to be personally accountable for the author's own contributions. $\mathrm{AH}$ had done the creation of new software used in the work and had approved the submitted version and had agreed both to be personally accountable for the author's own contributions.

\section{Funding}

The authors declare that they have no funding.

\section{Availability of data and materials}

The datasets used and/or analyzed during the current study are available from the corresponding author on reasonable request.

\section{Declarations}

\section{Ethics approval and consent to participate}

Declared in the "Methods" section: Ethical approval had been obtained from Medical Research Ethics Committee of Faculty of Medicine, Mansoura University (code no.: MD/138). Approval from Egyptian Ministry of Health was taken (no.: 1-2016/18). Patients signed their written consents after detailed explanation of the study protocol.

\section{Consent for publication}

Not applicable.

\section{Competing interests}

The authors declare that they have no competing interests.

\section{Author details}

${ }^{1}$ Chest Medicine Department, Mansoura Chest Hospital, Mansoura, Egypt. ${ }^{2}$ Chest Medicine Department, Faculty of Medicine, Mansoura University, Algomhoria Street, Mansoura City, Egypt. ${ }^{3}$ Clinical Pathology Department, Faculty of Medicine, Mansoura University, Mansoura, Egypt.

Received: 16 November 2020 Accepted: 5 April 2021

Published online: 15 April 2021

\section{References}

1. Morris A, Crothers K, Beck JM et al (2011) An Official ATS Workshop Report: emerging issues and current controversies in HIV-associated pulmonary diseases. ATS 8:17-26

2. Deniz G, Doroudi F, Tohme J et al (2016) HIV/AIDS: trends in the Middle East and North Africa region. Int J Infect Dis 44(2016):66-73

3. Lawn SD (2012) Point-of-care detection of lipoarabinomannan (LAM) in urine for diagnosis of HIV-associated tuberculosis: a state of the art review. BMC Infect Dis 12:103

4. WHO (2011) (b): Guidelines for intensified tuberculosis case finding and isoniazid preventive therapy for people living with HIV in resource constrained settings. Stop TB department. World Health Organization, Geneva

5. Schmidt SD, Mazzella MJ, Nixon RA et al (2012) A $\beta$ measurement by enzyme-linked immunosorbent assay. Methods Mol Biol 849:507-527

6. Swaminathan S, Padmapriyadarsini C, Narendran G (2010) HIV-associated tuberculosis: clinical update. Clin Infect Dis 50(10):1377-1386. https://doi. org/10.1086/652147

7. UNAIDS (The Joint United Nations Program on HIV/AIDS) (2016) Fact sheet November 2016, Global HIV statistics. the Joint United Nations Program on HIV/AIDS, UNAIDS 2016, Geneva

8. Sabin CA, Smith CJ, Youle M, Lampe FC, Bell DR, Puradiredja D, Lipman MCl, Bhagani S, Phillips AN, Johnson MA (2006) Deaths in the era of HAART: contribution of late presentation, treatment exposure, resistance and abnormal laboratory markers. AIDS 20(1):67-71. https://doi.org/10.1097/01.a ids.0000196178.73174.24

9. Swindells S, Komarow L, Tripathy S et al (2013) Screening for pulmonary tuberculosis in HIV-infected individuals: AIDS Clinical Trials Group Protocol A5253. Int J Tuberc Lung Dis 17(4):532-539

10. Were W, Moore D, Ekwaru P, Mwima G, Bunnell R, Kaharuza F, Rutherford G, Mermin J (2009) A simple screening tool for active tuberculosis in HIVinfected adults receiving antiretroviral treatment in Uganda. Int J Tuberc Lung Dis 13(1):47-53

11. Cobelens FG, Egwaga SM, van Ginkel T et al (2006) Tuberculin skin testing in patients with HIV infection: limited benefit of reduced cutoff values. Clin Infect Dis 43:634-639

12. Leeds IL, Magee MJ, Kurbatova EV et al (2012) Site of extrapulmonary tuberculosis is associated with HIV infection. Clin Infect Dis 55(1):75-81. https://doi.org/10.1093/cid/cis303

13. Kipp AM, Stout JE, Hamilton CD et al (2008) Extrapulmonary tuberculosis, human immunodeficiency virus, and foreign birth in North Carolina, 19932006. BMC Public Health 8:107

14. Tavs Q, Johansen IS, Pressler T et al (2014) Urine lipoarabinomannan pointof-care testing in patients affected by pulmonary nontuberculous mycobacteria - experiences from the Danish Cystic Fibrosis cohort study. BMC Infect Dis 14:655

15. Kroidl I, Clowes P, Mwakyelu J, Maboko L, Kiangi A, Rachow A, Reither K, Jung J, Nsojo A, Saathoff E, Hoelscher M (2013) Reasons for false-positive lipoarabinomannan ELISA results in a Tanzanian population. Scand J Infect Dis. 46(2):144-148. https://doi.org/10.3109/00365548.2013.853133

16. Peter JG, Green $C$, Hoelscher M et al (2010) Urine for the diagnosis of tuberculosis: current approaches, clinical applicability, and new developments. Curr Opin Pulm Med. 16:262-270

17. Cox JA, Lukande RL, Kalungi $S$ et al (2015) Is urinary lipoarabinomannan the result of renal tuberculosis? Assessment of the renal histology in an autopsy cohort of Ugandan HIV-infected adults. PLoS ONE 10(4):e0123323

18. Wood R, Lawn SD (2013) Challenges facing LAM urine antigen tests for diagnosing HIV associated tuberculosis. Expert Rev Mol Diagn. 12(6): 549-551

19. Lawn SD, Edwards DJ, Kranzer K et al (2009) Urine lipoarabinomannan assay for tuberculosis screening before antiretroviral therapy diagnostic yield and association with immune reconstitution disease. AIDS 23:1875-1880 
20. Lawn SD, Harries AD, Meintjes $G$ et al (2012) Reducing deaths from tuberculosis in antiretroviral treatment programmes in sub-Saharan Africa. AIDS 26:2121-2133

21. Lawn SD, Kerkhoff AD, Vogt M et al (2013) HIV-associated tuberculosis: relationship between disease severity and the sensitivity of new sputumbased and urine-based diagnostic assays. BMC Med 11(1):231. https://doi. org/10.1186/1741-7015-11-231

22. Drain PK, Gounder L, Sahid F et al (2016) Rapid urine LAM testing improves diagnosis of expectorated smear- negative pulmonary tuberculosis in an HIV-endemic region. Sci Rep. 6:19992

23. Peter JG, Theron G, Dheda K et al (2013) Can point-of-care urine LAM strip testing for tuberculosis add value to clinical decision making in hospitalised HIV-infected persons? PLoS ONE 8(2):e54875. https://doi.org/10.1371/journal. pone.0054875

24. Lydia N, Moodley M, Manabe YC et al (2014) Diagnostic accuracy of a rapid urine lipoarabinomannan test for tuberculosis in HIV-infected adults. AIDS 66(3):270-279

25. WHO (2015) (c): The use of lateral flow urine lipoarabinomannan assay (LFLAM) for the diagnosis and screening of active tuberculosis in people living with HIV. Policy guidance. World Health Organization, Geneva

26. Lester JF, Dojcinov SD, Attanoos RL et al (2003) The clinical impact of expert pathological review on lymphoma management: a regional experience. $\mathrm{Br} J$ Haematol 123(3):463-468. https://doi.org/10.1046/j.1365-2141.2003.04629.x

27. Maunank S, Martinson NA, Chaisson RE et al (2010) Quantitative analysis of a urine-based assay for detection of lipoarabinomannan in patients with tuberculosis. J Clin Microbiol 48(8):2972-2974

28. Maunank S, Variava E, Holmes CB et al (2009) Diagnostic accuracy of a urine lipoarabinomannan test for tuberculosis in hospitalized patients in a high HIV prevalence setting. AIDS 52:145-151

\section{Publisher's Note}

Springer Nature remains neutral with regard to jurisdictional claims in published maps and institutional affiliations.

\section{Submit your manuscript to a SpringerOpen ${ }^{\circ}$ journal and benefit from:}

- Convenient online submission

- Rigorous peer review

- Open access: articles freely available online

- High visibility within the field

- Retaining the copyright to your article

Submit your next manuscript at $\boldsymbol{\nabla}$ springeropen.com 